\title{
A Multi-Agent Control Architecture for Supply Chains using a Predictive Pull-Flow Perspective
}

\author{
J. Lemos Nabais ${ }^{1}$, Rudy R. Negenborn ${ }^{2}$, R.B. Carmona Benítez ${ }^{3}$, Luís F. Mendonça ${ }^{4}$, \\ João Lourenço $^{5}$, and M. Ayala Botto ${ }^{6}$ \\ 1 IDMEC, Department of Informatics and Systems Engineering \\ Setúbal School of Technology, Polytechnical Institute of Setúbal, Setúbal, Portugal \\ joao.nabais@estsetubal.ips.pt \\ 2 Delft University of Technology, Marine and Transport Technology \\ Transport Engineering and Logistics, Delft, The Netherlands \\ r.r.negenborn@tudelft.nl \\ ${ }^{3}$ School of Business and Economics, Universidad Anáhuac México Norte \\ Huixquilucan, México \\ rafael.carmona@anahuac.mx \\ 4 IDMEC, Escola Superior Naútica Infante D. Henrique \\ Department of Marine Engineering, Paço d'Arcos, Portugal \\ luismendonca@enautica.pt \\ 5 INESC, Department of Informatics and Systems Engineering \\ Setúbal School of Technology, Polytechnical Institute of Setúbal, Setúbal, Portugal \\ joao.lourencolestsetubal.ips.pt \\ ${ }^{6}$ IDMEC, Instituto Superior Técnico, Technical University of Lisbon, Department of \\ Mechanical Engineering, Lisboa, Portugal \\ ayalabottoeist.utl.pt
}

\begin{abstract}
Supply chains are large-scale distribution networks in which multiple types of commodities are present. In this paper, the operations management in supply chains is posed as a tracking control problem. All inventory levels in the network should be kept as close as possible to the desired values over time. The supply chain state is disturbed due to client demand at the end nodes. A multiagent control architecture to restore all inventory levels over the supply chain is proposed. First the model for the supply chain is broken down into smaller subsystems using a flow decomposition. The operations management for each subsystem will be decided upon by a dedicated control agent. The control agents solve their problems using a pull-flow perspective, starting at the end nodes and then propagating upstream. Adding new components to the supply chain will have as a consequence the inclusion of more control agents. The proposed architecture is easily scalable to large supply chains due to its modular feature. The multi-agent control architecture performance is illustrated using a supply chain composed of four levels (suppliers, consolidation, distribution, end nodes) using different levels of predictions about client demands. With the increase of prediction demand accuracy the proposed control architecture is able to keep the desired inventory level at the end nodes over time, which makes it suitable for use for just in time production strategies.
\end{abstract}

Keywords: supply chains, multi-agent systems, model predictive control, inventory level 


\section{Introduction}

Supply chain are complex systems in which multiple organizations (suppliers, manufacturers, retailers, and customers) are contributing to move commodities or services from a source node to a destination node [1]. The strong coupling between organizations restricts achieving optimal performance of the whole system. Currently, the increase of production far from the final customer poses a challenge to the existing supply chains. The problem is growing in complexity and new methodologies are required to support decisions leading to a more effective cooperation between organizations. This cooperation depends on the type of supply chain in terms of freedom to exchange information. In a vertical integration all organizations are owned by the same company and therefore the information can be shared freely. In horizontal integration different organizations are owned by different companies with possibly conflicting objectives and competitive issues, making the exchange information more restricted.

In this paper we consider client demand as an exogenous input at the end nodes that disturbs the supply chain state: inventory levels. Operations management is required to assign flows between nodes such that the client demand is satisfied while keeping the inventory at a desired level. This paper proposes a multi-agent control architecture to keep the desired inventory levels over the supply chain. As supply chains may be large-scale systems we propose a flow decomposition [2] to obtain smaller subsystems. A control agent is assigned to each subsystem and is responsible for determining decisions (flows assignment) over time. The control agent will solve an optimization problem at each time step in accordance to the Model Predictive Control (MPC) strategy. MPC has shown successful applications in the process industry [3], and is now gaining increasing attention in fields like supply chains [4], power networks [5], water distribution networks [6] and road traffic networks [7]. In supply chains, costs can be associated to flows and quantities of stored commodities. Using mathematical models to describe the flows inside supply chains it is possible to make predictions about the future behavior of the supply chain. The MPC controller can determine which actions have to be chosen in order to obtain the best performance. At each time step the controller first obtains the current state of the system it controls. Then it formulates an optimization problem, using the desired goals, existing constraints, disturbances and prediction information if available. The possibility to include prediction information in the optimization problem motivates the selection of this control strategy. Through this mechanism the different control agents can exchange information about their current and future decisions increasing their cooperation by avoiding multiple agents to answer to the same client demand. The order by which the control agents solve their problems is a so-called pull-flow perspective, starting from the end nodes, where the exogenous input is applied, and propagated towards the source nodes.

This paper is organized as follows. In Section 2 the model used for describing the multi-commodity flows in supply chains is given. The operations management problem is formulated in Section 3 and addressed using a multi-agent MPC architecture where each control agent solves an optimization problem at each discrete time step. The performance of the proposed architecture is tested through numerical experiments in Section 4 for a hypothetical supply chain taking into account different prediction accuracies. In Section 5 conclusions are drawn and future research topics are indicated. 


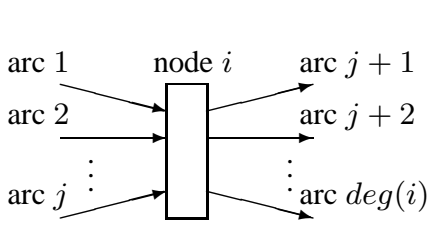

(a) Center node.

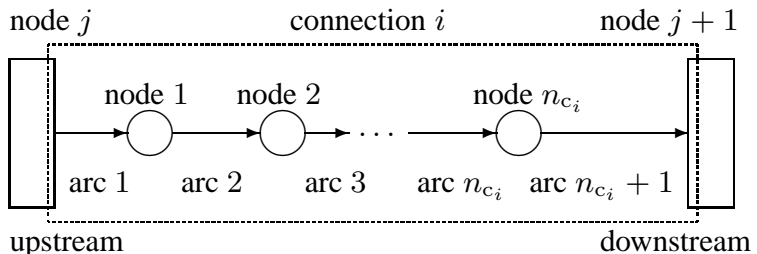

(b) Connection between two center nodes $(j$ and $j+1)$.

Fig. 1. Components in a supply chain to model the storage and transport phenomena $(\operatorname{deg}(i)$ stands for node $i$ degree).

\section{Modeling Supply Chains}

A supply chain is a network and can be described as a graph $\mathcal{G}=(\mathcal{V}, \mathcal{E})$ [8], where the nodes $\mathcal{V}$ are related to physical locations and the arcs $\mathcal{E}$ represent the available transport between nodes. At a macroscopic level, i.e., from a management perspective, supply chains exhibits two major phenomena: storage ability in well defined areas where commodities can be produced, manufactured or simply stored, and the transport delay (which is the time necessary to transport commodities between two nodes using the available transport). The storage ability is related to the so-called center nodes that can have multiple arriving and departing connections (see Fig. 1(a)). The transport delay for each connection is modeled as a succession of nodes with limited storage capacity (related to the transport capacity) between two center nodes (see Fig. 1(b)). Each connection has one upstream center node from where it pulls commodities, and it also has one downstream center node to where it pushes commodities. Connections can share the limited transport capacity to guarantee the desired flows between nodes. Supply chains are therefore complex systems with coupled dynamics and coupled constraints.

The complexity of the supply chain model is determined by:

- $n_{\mathrm{t}}$ : number of commodity types considered;

- $n_{\mathrm{c}}$ : number of connections existing in the supply chain;

- $n_{\mathrm{c}_{i}}$ : number of nodes belonging exclusively to connection $i$;

- $n_{\mathrm{n}}$ : number of center nodes in the supply chain that are further divided into source (upstream) nodes $n_{\mathrm{n}}^{\mathrm{u}}$, end (downstream) nodes $n_{\mathrm{n}}^{\mathrm{d}}$ and store nodes $n_{\mathrm{n}}^{\mathrm{s}}$;

- $n_{1}$ : number of levels present in the supply chain, including the source (upstream) and end node (downstream) levels.

For illustration purposes consider the supply chain represented in Fig. 2. The supply chain is divided into four levels $n_{1}=4$ (source, consolidation center, distribution center and end node levels) with a total of $n_{\mathrm{n}}=11$ center nodes connected through a total of $n_{\mathrm{c}}=17$ connections. The supply chain transports $n_{\mathrm{t}}=3$ commodities (products $\mathrm{A}, \mathrm{B}$ and C) generated at dedicated sources. As particular features the supply chain presents (1) the possibility to transport commodities between the distribution centers, (2) there are some end nodes that can be served by more than one connection and (3) available connections have different transport delays. 


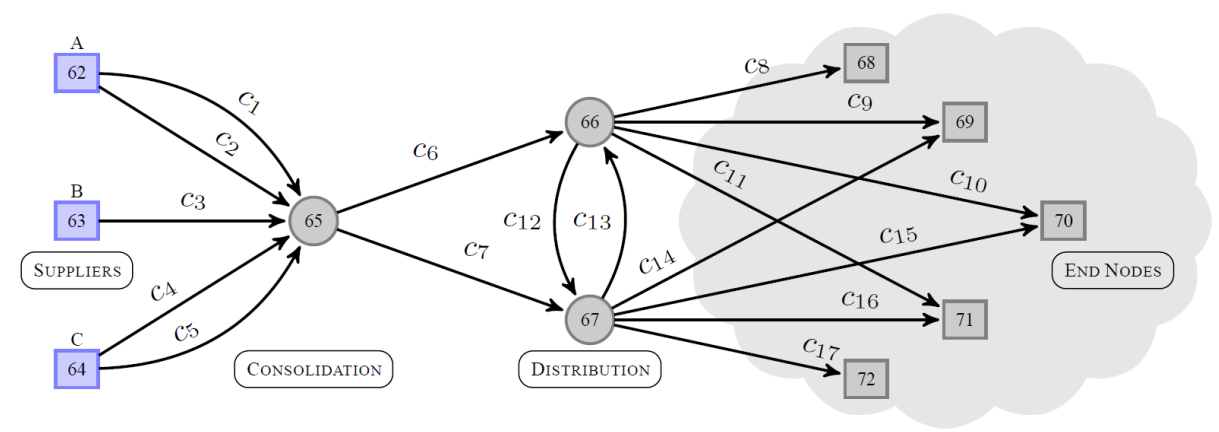

Fig. 2. Example of supply chain with three commodities, products A, B and C. For the sake of readability the 61 connection nodes are omitted.

\subsection{Proposed Centralized Dynamical Model}

The supply chain is seen as a network of storage areas described as queues that are connected by transport capacity represented by links. The proposed supply chain model for describing the supply chain dynamics is based on a flow perspective. The total number of nodes inside the supply chain $n_{\mathrm{y}}$ is associated with the network topology,

$$
n_{\mathrm{y}}=n_{\mathrm{n}}+\sum_{i=1}^{n_{\mathrm{c}}} n_{\mathrm{c}_{i}} .
$$

For each node in the supply chain a state-space vector $\overline{\mathbf{x}}_{j}(k)$ is defined; the individual state-space vectors of the nodes are merged to form the state-space vector $\mathbf{x}(k)$ of the complete supply chain,

$$
\overline{\mathbf{x}}_{j}(k)=\left[\begin{array}{c}
x_{j}^{1}(k) \\
x_{j}^{2}(k) \\
\vdots \\
x_{j}^{n_{\mathrm{t}}(k)}
\end{array}\right], j=1, \ldots, n_{\mathrm{y}}, \quad \mathbf{x}(k)=\left[\begin{array}{c}
\overline{\mathbf{x}}_{1}(k) \\
\overline{\mathbf{x}}_{2}(k) \\
\vdots \\
\overline{\mathbf{x}}_{n_{\mathrm{y}}}(k)
\end{array}\right]
$$

where $x_{j}^{t}(k)$ is the quantity per commodity type $t$ at node $j$ at time step $k$. The dimension of the state-space vector $\mathbf{x}(k)$ is given by $n_{\mathrm{t}} n_{\mathrm{y}}$. The model for the supply chain dynamics can now be represented in a compact form as

$$
\begin{aligned}
\mathbf{x}(k+1) & =\mathbf{A} \mathbf{x}(k)+\mathbf{B}_{\mathrm{u}} \mathbf{u}(k)+\mathbf{B}_{\mathrm{d}} \mathbf{d}(k) \\
\mathbf{y}(k) & =\mathbf{C} \mathbf{x}(k) \\
\mathbf{x}(k) & \geq \mathbf{0} \\
\mathbf{u}(k) & \geq \mathbf{0} \\
\mathbf{y}(k) & \leq \mathbf{y}_{\max } \\
\mathbf{P}_{\mathrm{uu}} \mathbf{u}(k) & \leq \mathbf{u}_{\max } \\
\mathbf{x}(k) & \geq \mathbf{P}_{\mathrm{xu}} \mathbf{u}(k)
\end{aligned}
$$


where $\mathbf{u}$ is the control action vector with length $n_{\mathrm{u}}=n_{\mathrm{t}}\left(n_{\mathrm{y}}-n_{\mathrm{n}}+n_{\mathrm{c}}\right)$ representing the flows between nodes, $\mathbf{d}$ is the exogenous input vector related to the downstream demand over time with dimension $n_{\mathrm{t}} n_{\mathrm{n}}^{\mathrm{d}}, \mathbf{y}$ is the current volume per commodity type at all nodes with dimension $n_{\mathrm{y}}, \mathbf{y}_{\text {max }}$ are the maximum node storage capacities, $\mathbf{u}_{\max }$ the maximum transport capacities according to the supply chain design, $\mathbf{A}, \mathbf{B}_{\mathrm{u}}, \mathbf{B}_{\mathrm{d}}$ and $\mathbf{C}$ are the state-space matrices, $\mathbf{P}_{\mathrm{xu}}$ is the projection from the control action set $\mathcal{U}$ into the state-space set $\mathcal{X}$ and $\mathbf{P}_{\text {uu }}$ is the projection matrix from the control action set $\mathcal{U}$ into the maximum transport capacity set $\mathcal{U}_{\max }$. The supply chain state, $\mathbf{x}$, at the next time step, $k+1$, is determined using (3) as a function of the current supply chain state, $\mathbf{x}(k)$, plus the control action contribution, $\mathbf{u}$, decided upon by the supply chain manager, and the corresponding exogenous inputs, $\mathbf{d}$, capturing the client demand. The control action $\mathbf{u}$ is the flow per commodity type between nodes and is imposed through a corresponding transport capacity allocation. Inequalities (5)-(9) are necessary in this framework for imposing the supply chain structural layout and assumptions made:

Nonnegativity of States and Flows: negative storage is not physically possible, imposed by (5), and all flows are assumed to be nonnegative, this is guaranteed by (6);

Storage Capacity: each node has to respect its own storage capacity and this is represented by (7);

Maximum Transport Capacity: the supply chain structural layout in terms of transport capacity is represented by (8);

Feasible Flows: not all flows that satisfy (5) and (6) are allowed. The assigned flow has to respect the quantity per commodity type available in the related node and therefore equation (9) constraints this relation.

\subsection{Supply Chain Decomposition}

Real supply chains may consist of tens of center nodes and handle hundreds of commodity types. It is critical to alleviate the computational burden introduced when considering the central model (3)-(9) such that a solution is reached in admissible time. Using a node/arc numbering in a push-flow perspective (from the sources towards the end nodes) it is possible to obtain a highly structured state-space model without the need to further mathematical manipulations [9]. Although the network is composed of several center nodes it is important to note that a connection is by definition the path between two center nodes. Therefore the interference of a single connection into the set of center nodes is done solely at two nodes. A subsystem $i$ is defined as the node collection related to a connection $i$ plus the associated source and end nodes [2]. The state-space vector $\mathbf{x}_{i}$ for subsystem $i$ will be composed of the corresponding $\overline{\mathbf{x}}_{j}$ state-space vectors,

$$
\mathbf{x}_{i}(k)=\left[\begin{array}{c}
\overline{\mathbf{x}}_{n_{\mathrm{C}_{i}}-n_{\mathrm{c}_{i}}+1}(k) \\
\overline{\mathbf{x}}_{n_{\mathrm{C}_{i}}-n_{\mathrm{c}_{i}}+2}(k) \\
\vdots \\
\overline{\mathbf{x}}_{n_{\mathrm{C}_{i}}-1}(k) \\
\overline{\mathbf{x}}_{n_{\mathrm{C}_{i}}}(k) \\
\overline{\mathbf{x}}_{i}^{\text {in }}(k) \\
\overline{\mathbf{x}}_{i}^{\text {out }}(k)
\end{array}\right], n_{\mathrm{C}_{i}}=\sum_{j=1}^{i}\left(n_{\mathrm{c}_{j}}\right), \quad 1 \leq i \leq n_{\mathrm{c}}
$$


with length $n_{\mathrm{t}}\left(n_{\mathrm{c}_{i}}+2\right)$ belonging to state-space set $\mathcal{X}_{i}$ where $\overline{\mathbf{x}}_{i}^{\text {in }}$ and $\overline{\mathbf{x}}_{i}^{\text {out }}$ are the state-space vectors related to the source and end nodes for connection $i$ respectively. The state-space model for subsystem $i$ is given by,

$$
\begin{aligned}
\mathbf{x}_{i}(k+1) & =\mathbf{A}_{i} \mathbf{x}_{i}(k)+\mathbf{B}_{\mathrm{u}_{i}} \mathbf{u}_{i}(k)+\mathbf{B}_{\mathrm{d}_{i}} \mathbf{d}_{i}(k)+\sum_{j=1, j \neq i}^{n_{\mathrm{c}}} \mathbf{B}_{\mathrm{u}_{i, j}} \mathbf{u}_{j}(k) \\
\mathbf{y}_{i}(k) & =\mathbf{C}_{i} \mathbf{x}_{i}(k),
\end{aligned}
$$

where $\mathbf{y}_{i}$ is the total quantity of commodities at subsystem $i$ nodes, $\mathbf{u}_{i}$ is the control action for subsystem $i$ with length $n_{\mathrm{t}}\left(n_{\mathrm{c}_{i}}+1\right)$ belonging to set $\mathcal{U}_{i}, \mathbf{d}_{i}$ is the exogenous input vector associated with subsystem $i, \mathbf{A}_{i}, \mathbf{B}_{\mathrm{u}_{i}}, \mathbf{B}_{\mathrm{u}_{i, j}} \mathbf{B}_{\mathrm{d}_{i}}$ and $\mathbf{C}_{i}$ are the statespace matrices for subsystem $i$. The last term in (11) is responsible for the information exchange between control agents, in particular regarding their future behavior, to avoid that two or more control agents respond to the same demand. The complete subsystem $i$ model is obtained including constraints of nonnegativity of states and flows, storage capacity, maximum transport capacity, and feasible flows to the state space (11)-(12).

\section{Supply Chain Operations Management}

To limit the problem dimension to be solved at each time step, a control agent is assigned to each subsystem obtained from the supply chain using the flow decomposition of the previous section. This approach leads to a scalable and modular control architecture. Adding new connections and nodes has as consequence the inclusion of more control agents. In order to assure the cooperation between the different control agents it is critical to assure or promote information exchange between control agents regarding their current and predicted future decisions to avoid multiple agents to answer to the same client demand. Control agents solve their problems, one after another, using the information of the previous control agent but no communication iterations are performed between control agents [10].

\subsection{MPC Formulation For One Control Agent}

Control agent $i$ will solve the operations management for subsystem $i$ following an MPC strategy. MPC is an online optimization-based control approach that minimizes an objective function subject to constraints at each time step.

The solution to the optimization problem is an optimal sequence of control actions over the prediction horizon that give the best predicted performance. The controller implements only the component corresponding to the first time step until the beginning of the next time step, in a receding horizon fashion. At the next time step the MPC controller searches for the solution of a new optimization problem, i.e., by obtaining new information about the current state, available prediction information, and goals.

The cost function of a control agent is defined in accordance to the application field and it is generally a function of the subsystem states and control actions that the agent 
controls over the prediction horizon $N_{\mathrm{p}}$,

$$
J_{i}\left(\tilde{\mathbf{x}}_{k, i}, \tilde{\mathbf{u}}_{k, i}, \tilde{\mathbf{x}}_{\mathrm{ref}, i}\right)=\sum_{l=0}^{N_{\mathrm{p}}-1} f\left(\mathbf{x}_{i}(k+1+l), \mathbf{u}_{i}(k+l), \mathbf{x}_{\mathrm{ref}, i}(k)\right),
$$

where $\tilde{\mathbf{x}}_{k, i}$ is the vector composed of the state-space vectors for each time step over the prediction horizon $\left[\mathbf{x}_{i}^{\mathrm{T}}(k+1), \ldots, \mathbf{x}_{i}^{\mathrm{T}}\left(k+N_{\mathrm{p}}\right)\right]^{\mathrm{T}}, \tilde{\mathbf{u}}_{k, i}$ is the vector composed of the control action vectors for each time step over the prediction horizon $\left[\mathbf{u}_{i}^{\mathrm{T}}(k), \ldots, \mathbf{u}_{i}^{\mathrm{T}}\left(k+N_{\mathrm{p}}-1\right)\right]^{\mathrm{T}}, \mathbf{x}_{\mathrm{ref}, i}$ is the desired inventory level vector, $\tilde{\mathbf{x}}_{\text {ref }, i}$ is the vector composed of the desired inventory level vectors for each time step over the prediction horizon $\left[\mathbf{x}_{r e f, i}^{\mathrm{T}}(k), \ldots, \mathbf{x}_{r e f, i}^{\mathrm{T}}\left(k+N_{\mathrm{p}}-1\right)\right]^{\mathrm{T}}$ for control agent $i$. The MPC formulation for control agent $i$ can be stated as:

$$
\min _{\tilde{\mathbf{u}}_{k, i}} J_{i}\left(\tilde{\mathbf{x}}_{k, i}, \tilde{\mathbf{u}}_{k, i}, \tilde{\mathbf{x}}_{\mathrm{ref}, i}\right)
$$

subject to $\mathbf{x}_{i}(k+1+l)=\mathbf{A}_{i} \mathbf{x}_{i}(k+l)+\mathbf{B}_{\mathbf{u}_{i}} \mathbf{u}_{i}(k+l)+\mathbf{B}_{\mathrm{d}_{i}} \mathbf{d}_{i}(k+l)+\ldots$

$$
\begin{aligned}
& \ldots+\sum_{j=1, j \neq i}^{n_{\mathrm{c}}} \mathbf{B}_{\mathbf{u}_{i, j}} \mathbf{u}_{j}(k+l), \\
& \mathbf{y}_{i}(k)=\mathbf{C}_{i} \mathbf{x}_{i}(k), \\
& \mathbf{x}_{i}(k+1+l) \geq \mathbf{0}, \\
& \mathbf{u}_{i}(k+l) \geq \mathbf{0}, \\
& \mathbf{y}_{i}(k+l) \leq \mathbf{y}_{\max , i}, \\
& \mathbf{P}_{\mathrm{uu}, i} \mathbf{u}_{i}(k+l) \leq \mathbf{u}_{\max , i}, \\
& \mathbf{x}_{i}(k+l) \geq \mathbf{P}_{\mathbf{x u}, i} \mathbf{u}_{i}(k+l),
\end{aligned}
$$

where $\mathbf{y}_{\max , i}$ is the maximum capacity for the nodes of control agent $i, \mathbf{u}_{\max , i}$ represents the available transport resources according to the network's structural layout for control agent $i, \mathbf{P}_{\mathrm{uu}, i}$ is the projection matrix from the control action set $\mathcal{U}_{i}$ into the transport resource set for control agent $i, \mathbf{P}_{\mathrm{xu}, i}$ is the projection from the control action set $\mathcal{U}_{i}$ into the state-space set $\mathcal{X}_{i}$.

\subsection{Multi-Agent Control Architecture}

The order in which the control agents solve their problems at each time step can be fixed over time or depend on the current supply chain state and predictions. For the sake of simplicity we consider that the order $\mathbf{o}(k)=\left[o_{1} \ldots o_{n_{\mathrm{c}}}\right]$, with $1 \leq o_{i} \leq n_{\mathrm{c}}$, by which the control agents solve their problems is fixed over time and is a supply chain configuration parameter set before the beginning of operations management. The order of control agents is set following a pull-flow perspective: first the control agents related to the end nodes solve their problems to keep the desired inventory levels. This will pull commodities from the distribution center level. Then the control agents related to the distribution centers solve their problems, pulling commodities from the consolidation level and so on, until the control agents that pull commodities from the source nodes.

The starting control agent is responsible for setting the total amount of transport capacity that is available $\theta^{0}=\mathbf{u}_{\max }$ for the current time step and the current prediction 


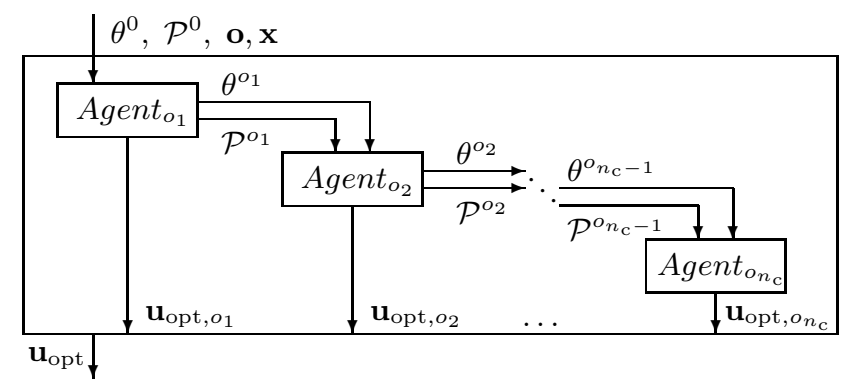

Fig. 3. Multi-agent control architecture schematics at a time step.

for future decisions set $\mathcal{P}^{0}=\left\{\tilde{\mathbf{u}}_{k-1, o_{1}}, \ldots, \tilde{\mathbf{u}}_{k-1, o_{n_{c}}}\right\}$. After the initial configuration iterations are executed. The starting control agent $\left(o_{1}\right)$ has all transport capacity available. Each control agent $o_{i}\left(i=1, \ldots, n_{\mathrm{c}}\right)$, one after another, performs the following steps at an iteration (see Fig. 3):

- the maximum admissible transport capacity for control agent $o_{i}$ is determined as the minimum between the subsystem maximum transport capacity $\mathbf{u}_{\max , o_{i}}$ and the transport capacity not yet assigned,

$$
\mathbf{u}_{\max , o_{i}}=\min \left(\mathbf{P}_{\max , o_{i}} \theta^{o_{i-1}} ; \mathbf{u}_{\max , o_{i}}\right),
$$

where $\mathbf{P}_{\max , o_{i}}$ is the projection matrix from the global transport capacity set $\mathcal{U}_{\max }$ to the maximum transport capacity set $\mathcal{U}_{\max , o_{i}}$ for subsystem $o_{i}$;

- the optimal control action $\mathbf{u}_{\mathrm{opt}, o_{i}}$ is the first time step component of the control agent optimal sequence over the prediction horizon $\tilde{\mathbf{u}}_{\mathrm{opt}, o_{i}}$ found solving the MPC problem (14)-(21) for control agent $i$;

- the available transport capacity to the next control agent $o_{i+1}$ is updated:

$$
\theta^{o_{i+1}}=\theta^{o_{i}}-\mathbf{P}_{\mathrm{mu}, o_{i}}(k) \mathbf{u}_{\mathrm{opt}, o_{i}}(k),
$$

where $\mathbf{P}_{\mathrm{mu}, o_{i}}(k)$ is the projection matrix from control agent $o_{i}$ handling resource set $\mathcal{U}_{o_{i}}$ to the control action set $\mathcal{U}_{\max }$;

- the predictions for future decisions are updated and denoted by $\mathcal{P}^{o_{i+1}}$ replacing the control agent initial prediction $\tilde{\mathbf{u}}_{k-1, o_{i}}$ by the new optimal sequence found $\tilde{\mathbf{u}}_{\mathrm{opt}, o_{i}}$.

\section{Simulation Experiments}

The proposed architecture is applied to the supply chain presented in Fig. 2. We focus on addressing the supply chain operations management as a flow assignment problem using the multi-agent architecture presented in Section 3. The structural design of the supply chain is out of the scope of this paper. For the sake of clarity we consider constant inventory levels over time. The performance obtained with the multi-agent architecture will be evaluated for three different configurations concerning the prediction accuracy available to the control agents: exact prediction (Test A), constant prediction (Test B) and no prediction (Test C). 


\begin{tabular}{||l||r|r|r|r|r||r|r||r|r|r|r|r||r|r|r|r|r||}
\hline \hline Parameters & $c_{1}$ & $c_{2}$ & $c_{3}$ & $c_{4}$ & $c_{5}$ & $c_{6}$ & $c_{7}$ & $c_{8}$ & $c_{9}$ & $c_{10}$ & $c_{11}$ & $c_{12}$ & $c_{13}$ & $c_{14}$ & $c_{15}$ & $c_{16}$ & $c_{17}$ \\
\hline Transport [hours] & 14 & 8 & 8 & 8 & 14 & 8 & 8 & 6 & 8 & 12 & 10 & 8 & 8 & 10 & 12 & 8 & 6 \\
\hline Source node & 62 & 62 & 63 & 64 & 64 & 65 & 65 & 66 & 66 & 66 & 66 & 66 & 67 & 67 & 67 & 67 & 67 \\
End node & 65 & 65 & 65 & 65 & 65 & 66 & 67 & 68 & 69 & 70 & 71 & 67 & 66 & 69 & 70 & 71 & 72 \\
\hline Nodes $\left(n_{\mathrm{c}_{i}}\right)$ & 6 & 3 & 3 & 3 & 6 & 3 & 3 & 2 & 3 & 5 & 4 & 3 & 3 & 4 & 5 & 3 & 2 \\
Flows & 7 & 4 & 4 & 4 & 7 & 4 & 4 & 3 & 4 & 6 & 5 & 4 & 4 & 5 & 6 & 4 & 3 \\
\hline Transport cost & 1 & 5 & 5 & 5 & 1 & 5 & 5 & 5 & 5 & 5 & 5 & 5 & 5 & 5 & 5 & 5 & 5 \\
Transport capacity & 260 & 100 & 100 & 100 & 260 & 80 & 80 & 30 & 30 & 30 & 30 & 10 & 10 & 30 & 30 & 30 & 30 \\
\hline \hline
\end{tabular}

Table 1. Connection details for the considered supply chain.

\begin{tabular}{||l||r|r|r|r|r||}
\hline \hline Commodity & end node 68 & end node 69 & end node 70 & end node 71 & end node 72 \\
\hline \hline A & 7.0 & 9.8 & 12.6 & 9.8 & 7.0 \\
B & 8.4 & 8.4 & 11.2 & 1.2 & 5.6 \\
C & 5.6 & 7.0 & 9.8 & 8.4 & 4.2 \\
\hline Total & 15 & 18 & 24 & 21 & 12 \\
\hline \hline
\end{tabular}

Table 2. Supply chain average demand for end nodes (quantity per time step).

\subsection{Setup}

The supply chain monitoring and management decision update is done every 2 hours. All supply chain nodes work on a 24 hour daily basis. The end nodes are opened to clients from $8 \mathrm{am}$ to $10 \mathrm{pm}$. The first disturbance will be available at $10 \mathrm{am}$ translating the consumption per commodity type between $8 \mathrm{am}$ and $10 \mathrm{am}$. The supply chain can be delivering commodities to supermarkets or raw materials to industries for example.

Using 2 hours as time step size, the transport delay per connection is translated into the required number of nodes to capture this phenomena (see Table 1). The supply chain model has 61 nodes to capture the transport delay for all connections: transport delays are assumed fixed. For end nodes 69,70 , and 71 commodities can be delivered from both distribution centers using a master connection (less transport time) or a slave connection (higher transport time). The supply chain demand is created as a random demand per time step for all commodities at the five end nodes (center nodes 68 to 72 , for average values see Table 2). The inventory levels are set to support the associated average demand during two, three and two complete days for the end nodes, distribution centers, and consolidation center respectively. To increase the demand challenge applied to the supply chain two demand peaks are set: one at the fourth day (a factor of 1.5) and one at the eight day (a factor of 2).

Control agent $i$ is assigned to connection $i$. All control agents solve the MPC problem using a prediction horizon of 7 steps corresponding to the biggest connection transport delay at the supply chain. As a cost function a linear penalty for deviations from the desired inventory level and transport costs is used. The state weights for the objective function are set in a pull perspective; in that sense the benefit for staying at a downstream node has to be bigger than the benefit staying at an upstream node. The order by which the control agents solve their problems is the following: $c_{15}, c_{10}, c_{16}$, $c_{11}, c_{9}, c_{14}, c_{17}, c_{8}, c_{6}, c_{13}, c_{7}, c_{12}, c_{1}, c_{2}, c_{3}, c_{5}$ and $c_{4}$. When multiple connections 


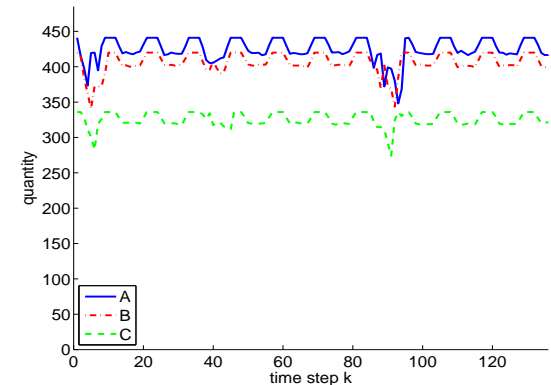

(a) Inventory level at node 67 .

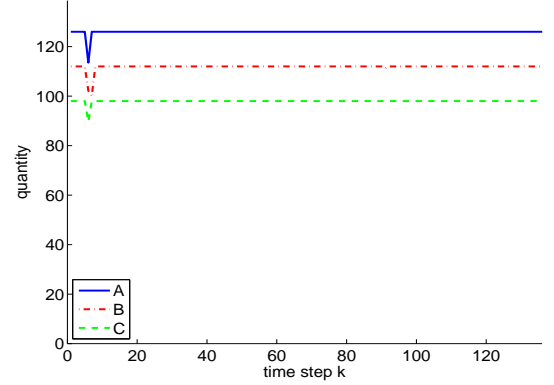

(b) Inventory level at node 70 .

Fig. 4. Inventory levels for exact demand prediction (test A).

arrive at the same center node priority was given to the closest or to the cheapest connection. The multi-agent architecture is solved at each time step of the simulation using the MPT v2.6.3 toolbox [11] with the CDD Criss-Cross solver for linear programming problems. The simulations are performed using MatLab R2009b on a personal computer with a processor Intel(R) Core(TM) i7 at $1.60 \mathrm{GHz}$ with 8 GB RAM memory in a 64-bit Operating System.

\subsection{Results Analysis}

The computational burden can be associated to the control action matrices $\mathbf{B}_{u}$ and $\mathbf{B}_{u_{i}}$. Using the proposed decomposition it is possible to reduce the matrix dimension from 50544 elements to 2736 , this is a reduction of $94.4 \%$. Naturally the ratio of nonzero elements grows from 0.009 to 0.171 . For test $\mathrm{A}$, the average computation time for each time step was $27.04 \mathrm{~s}$, with a maximum time of $40.8 \mathrm{~s}$ and a minimum time of $17.1 \mathrm{~s}$.

Increasing the accuracy of the available demand prediction the multi-agent architecture is able to keep the desired inventory levels at the end nodes, see Fig. 4(b). The architecture uses the available prediction to anticipate future events and start to move commodities in advance. Although the inventory level at the end nodes remain constant the other nodes face variation in their inventory levels (see Fig. 4(a)). With an accuracy decrease on the demand prediction the control agents do not have the necessary information to anticipate correctly the future demand. As a consequence the inventory levels at the end nodes start to face bigger oscillations and can run out of stock (see Fig. 5). As is to be expected, the average deviation from the initial inventory level is smaller for control agents that use exact demand prediction and is bigger for the case of no demand prediction (see Table 3). Due to the supply chain structural design all demand predictions show significant deviation at end node 68 . The exact demand prediction concentrates all deviation in commodity $C$. End node 70 has the worst indicators among the exact demand prediction which is justified by the higher demand and transport delay from the distribution centers associated.

Fig. 6 shows the state evolution for connection 10 which is the slave connection for node 71. Commodities are only dispatched from the connection source node if they are 


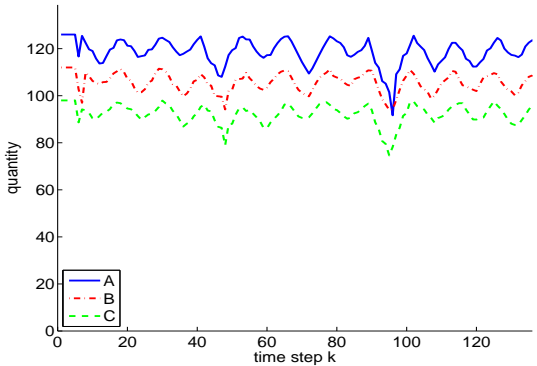

(a) Test B.

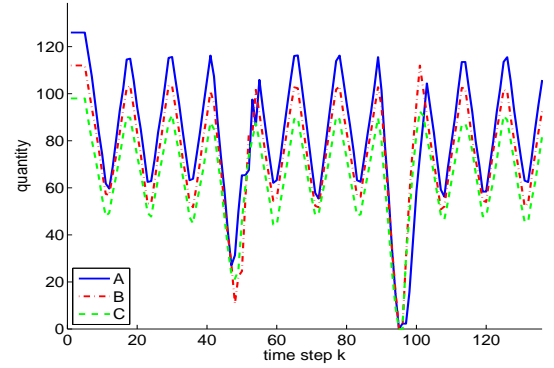

(b) Test C.

Fig. 5. Inventory levels at end node 70.

\begin{tabular}{||l|r|r|r|r|r||}
\hline \hline Deviation & end node 68 & end node 69 & end node 70 & end node 71 & end node 72 \\
\hline \hline $\max _{A}$ & $0.0 / 9.5 / 39.5$ & $0 / 26.8 / 68.0$ & $12.5 / 34.3 / \mathbf{1 2 6 . 0}$ & $0.0 / 39.0 / 67.5$ & $0.0 / 5.3 / 34.7$ \\
$\max _{B}$ & $0.0 / 31.7 / 73.6$ & $0 / 12.1 / \mathbf{8 4 . 0}$ & $11.8 / 18.0 / \mathbf{1 1 2 . 0}$ & $0.0 / 28.6 / \mathbf{1 1 2 . 0}$ & $0.0 / 6.2 / 30.2$ \\
$\max _{C}$ & $46.0 / 17.1 / 33.6$ & $0 / 14.8 / 62.1$ & $8.3 / 23.4 / \mathbf{9 8 . 0}$ & $0.0 / 25.0 / 78.0$ & $2.1 / 4.3 / 21.7$ \\
\hline $\operatorname{mean}_{A}$ & $0.0 / 2.2 / 11.8$ & $0.0 / 3.6 / 22.4$ & $0.1 / 7.4 / 43.5$ & $0.0 / 4.2 / 22.4$ & $0.0 / 1.8 / 11.5$ \\
$\operatorname{mean}_{B}$ & $0.0 / 2.8 / 15.5$ & $0.0 / 3.3 / 21.0$ & $0.2 / 6.5 / 37.1$ & $0.0 / 4.1 / 27.3$ & $0.0 / 1.5 / 9.2$ \\
$\operatorname{mean}_{C}$ & $1.5 / 1.9 / 9.7$ & $0.0 / 2.8 / 16.1$ & $0.1 / 5.8 / 32.3$ & $0.0 / 3.6 / 21.4$ & $0.0 / 1.2 / 7.0$ \\
\hline \hline
\end{tabular}

Table 3. Inventory analysis for the entire simulation time (exact/close/none demand prediction), bold values stands for out of stock.

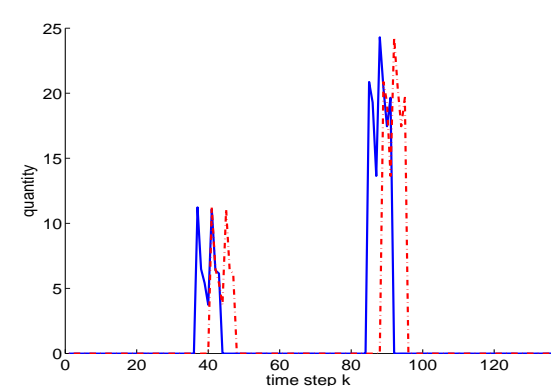

(a) Test A.

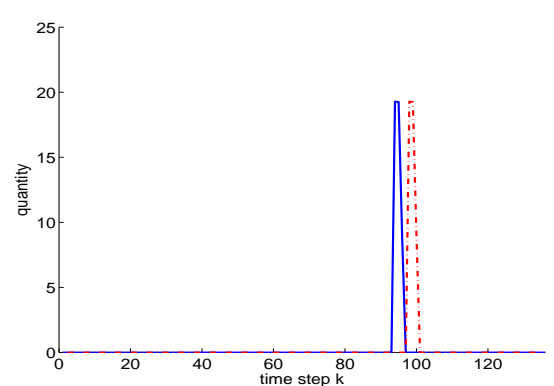

(b) Test C.

Fig. 6. Quantity of commodities at first and last nodes for connection 10 (full line: $\overline{\mathbf{x}}_{i, 1}$, dash-dot line: $\overline{\mathbf{x}}_{i, n_{\mathrm{c}_{i}}}$ ).

guaranteed to be accepted at the connection end node. There is no waiting queue at the connection. Decreasing the accuracy in demand prediction makes the slave connection to transports a lower volume of commodities leading to the decrease of inventory levels at the end node. For exact prediction, commodities are delivered at node 70 using the master connection with the ratios $1.00,0.95,0.77$ for commodities of type A, B, and $\mathrm{C}$ respectively. As no distinguish is made in terms of commodities the slave connection has a higher impact for the last commodity type. 


\section{Conclusions and Future Research}

The tracking control problem for multi-commodity supply chains has been addressed in this paper through a multi-agent control architecture using a pull-flow perspective. When the demand prediction is accurate the control architecture is able to continuously restore the inventory levels at the end nodes. This is the case in which the supply chain is delivering commodities to clients that know their demands in advance. For situations in which the demand is unknown by nature (as in the case of supermarkets) the control architecture performance will be depending on the prediction accuracy.

In future research the proposed control architecture will be extended to consider the inventory levels as a decision variable in the optimization problem. The question is to set the best level of inventories over the supply chain such that the demand is still fulfilled while minimizing storage costs. The case in which the supply chain is composed by distinct economic actors will also be considered. In this case, the information exchange between control agents is restricted and they may give conflicting objectives. Therefore, negotiation between control agents has to be included into the proposed architecture.

\section{Acknowledgements}

This research is supported by the VENI project "Intelligent multi-agent control for flexible coordination of transport hubs" (project 11210) of the Dutch Technology Foundation STW, a subdivision of the Netherlands Organisation for Scientific Research (NWO) and by the Portuguese Government, through Fundação para a Ciência e a Tecnologia, under the project PTDC/EEACRO/102 102/2008 - AQUANET, through IDMEC under LAETA.

\section{References}

1. Ballou, R.H.: Business Logistics - Supply Chain Management: Planning, Organizing, and Controlling the Supply Chain. Prentice Hall (2004)

2. Nabais, J.L., Negenborn, R.R., Botto, M.A.: Hierarchical Model Predictive Control for Optimizing Intermodal Container Terminal Operations, submitted to a conference (2012)

3. Maciejowski, J.M.: Predictive control with constraints. Harlow, UK: Prentice Hall (2002)

4. Maestre, J.M., Muñoz de la Pena, D., Camacho, E.F.: Distributed MPC: a supply chain case. 48th IEEE Conference on Decision and Control and 28th Chinese Control Conference, pp. 7099-7104, Shanghai, China, December (2009)

5. Geyer, T., Larsson, M., Morari, M.: Hybrid emergency voltage control in power systems. In: Proceedings of the European Control Conference. Cambridge, UK. paper 322 (2003)

6. Negenborn, R.R., Van Overloop, P.J., Keviczky, T., De Schutter, B.: Distributed model predictive control of irrigation canals. Networks and Heterogeneous Media 4 359-380 (2009)

7. Hegyi, A., De Schutter, B., Hellendoorn, J.: Optimal coordination of variable speed limits to supress schock waves. IEEE Trans. Intelligent Transportation Systems 11 (1) 102-112 (2005)

8. Ahuja, R.K., Magnanti, T.L., Orlin, J.B.: Network Flows. New Jersey: Prentice Hall (1993)

9. Sezer, M.E., Šiljak, D.D.: Decentralized Control, The Control Handbook, William S. Levine Eds., CRC Press, pp. 779-793 (1996)

10. Negenborn, R.R., Sahin, A., Lukszo, Z., De Schutter, B., Morari, M.: A non-iterative cascaded predictive control approach for control of irrigation canals. In Proc. IEEE Int. Conf. on Systems, Man, and Cybernetics, pp.3652-3657, San Antonio, Texas, October (2009)

11. Kvasnica, M., Grieder, P., Baotić, M.: Multi-parametric toolbox (MPT), http://control.ee.ethz.ch/ mpt/ (2004) 